\title{
GENERAL PROPERTIES OF ULTRAVIOLET FLARES IN RS CVn SYSTEMS
}

\author{
J. E. NEFF', A. BROWN ${ }^{2}$, and J. L. LINSKY' \\ 'NASA Goddard Space Flight Center \\ ${ }^{2}$ JILA, U. of Colorado and NBS
}

\begin{abstract}
Approximately 10 very bright flares in RS CV'n systems have been observed with the IUE. In several cases, high-resolution spectra of the $\mathrm{Mg}$ II $\mathrm{k}$ line profiles were obtained before, during, and after the flare. Such data permit us to measure the position and size of the flaring region, the amount of line broadening, and any systematic fluw velocity. In two cases, several spectra were obtained during the flare decay, permitting us to study the change in these quantities during the flare decay.
\end{abstract}

\section{The 19 September 1985 Flare on AR Lac}

During 18-19 September 1985, we observed AR Lac continuously with IUE for $40 \mathrm{~h}$, obtaining a series of high-resolution LWP spectra of the $\mathrm{Mg}$ II k (2795.5 $\AA$ ) line. A low-resolution SWP spectrum of the 1200 to $1000 \AA$ region was obtained following each LWP spectrum. The high-resolution spectra were used to construct images of the chromospheres (Neff 1987; Neft et al. 1988a) of both stars in the system. The spectrum obtained at 1510 UT on 19 September showed a dramatic increase in the flux of the $\mathrm{Mg}$ II $\mathrm{k}$ emission fiom the $\mathrm{G}$ star compared to previous spectra. By subtracting the mean enission line protile of the $G$ star prior to this flare from this and subsequent spectra, the emission from the flatre alone was isolated. The decay in flux and width of the flare $\mathrm{Mg}$ II $\mathrm{k}$ emission is shown in Figure 1.

We estimated the maximum area of the flaring region (1 to $3 \%$ of the visilste hemisphere) from the line width in the latest decay phases by assuming that the: width is due only to rotational smearing and that the region is circular. The line was $60 \mathrm{~km} \mathrm{~s}^{-1}$ broarler near flare peak, due to an additional broadening mechanism.

The peak $\mathrm{Mg}$ II $\mathrm{k}$ surface flux in the flare was $\geq 2.7 \times 10^{8} \mathrm{erg} \mathrm{s}^{-1} \mathrm{~cm}^{-2}$, nearly 100 times greater than the mean non-flaring level for the $\mathrm{G}$ star. The rise time to peak flux wais $<2.4 \mathrm{~h}$, and the $1 / \mathrm{e}$ decay time scale at $\mathrm{Mg}$ II $\mathrm{k}$ was $3.5 \mathrm{~h}$. The minimum total radiative energy output in the $\mathrm{Mg}$ II $\mathrm{k}$ line alone during the flare was $\geq 2.5 \times 10^{31} \mathrm{erg}$.

At flare peak, the measured radial velocity of the flare con,ponent (Figure 2) was redshifted by $\sim 25 \mathrm{~km} \mathrm{~s}^{-1}$ with respect to the radial velocity of the $G$ star. Near the cnd of the flare, the measured relative velocity was $\sim 10 \mathrm{~km} \mathrm{~s}^{-1}$ redward of line center. The rotation of a discrete region would cause its emission profile to move $\sim 15 \mathrm{~km} \mathrm{~s}^{-1}$ from blue to red during this phase interval. There was therefore a systematic red ihlift of the flare emission of $\sim 30 \mathrm{~km} \mathrm{~s}^{-1}$ ncar the flare peak.

\section{The 3 October 1981 Flare on V 711 Tau}

The analysis of the higl-resolution spectra obtained during the 3 October 1981 flare on V711 Tau (Linsky et al. 1988) was performed in the same fashion as used for the AR Lac 
flare. By subtracting the pre flace profile from those during the flare, we were able to recover the $\mathrm{Mg}$ II $\mathrm{k}$ profile of the flare near its peak.

Near flatre peak, the fliare enission was broad $\left(\sim 60 \mathrm{~km} \mathrm{~s}^{-1}\right)$ and was centered at $90 \pm 30$ kin $\mathrm{s}^{-1}$ relative to the central meridian of the $K$ star. This velocity shift is the sum of the projected rotational velocity of the flaring region and of systematic flows. Since the projected rotational velocity of the $K$ star is $40 \mathrm{~km} \mathrm{~s} \mathrm{~s}^{-1}$, the dowuftow velocity would be $50 \mathrm{~km} \mathrm{~s}^{-1}$ if the flare were lerated uear the receeding limb of the $\mathrm{K}$ star, or larger if it were at other positions on the star. The density- sensitive ratio of SillI](1892 $\AA$ )/CIII](1908 $\AA$ ) changed radically cluring, the flawe, permitting us to measure the mean density of the flaring plasma.

\section{Other Ultraviolet Flares Observed with IUE}

\subsection{AlR Liac}

Walter et al. (1987) reported on the 5 October 1983 flare on AR Lac. A radio flare was cheserved at nearly the same time: with the VLA. By subtracting the pre-flare of star profile firme the observed profile, they determined the flux and width of the flare near flare peak. 'l'he unasured wirlth of the flare profile corresponded to $85 \mathrm{~km} \mathrm{~s}^{-1}$. Unfortunately', the pliase arratge of this data set was relatively poor, so it also is possible to interpret the excess "unission as rotational modulation of the profile due to plages on the stellar surface. The asserediation with the radio flare is therefore inconclusive.

An intentse: ultraviolet flare on AR Lac was observed on 31 August 1984 (Walter et al. 1984). Unfortunately, only low-resolution observations were obtained. As fate would have it, the simultancous observing campaign at other wavelengths did not begin until the following liay.

\subsection{IID 199178}

Fon or perhaps five flates have becn observed on HD 199178 (which has been classified as an "FK Comate" star). Three of these flares occurred at approxinately the same photometric fhatse (sce Netf, Vilhu, and Walter 1988b). On 19 September 1987, the overall Mg II k profile was observed to brighten and broaden considerably, but only one spectrum was obtained during the llare.

We obtrined two high-resolution spectra of the 6 September 1986 flare. Unfortunately, 110) low-resulution SWP spectra w... obtined during the flare. No increase in broading was seen in the two flare spectra.

\section{$3.3 \mathrm{UX}$ Ari}

Sinon, linsky, and Schiffer (19S0) reported on the 1 January 1979 flare of UX Ari. The $\mathrm{Mg}$ II line luring the flare was strongly asymmetric, with the red wing of the line blending with the Mg II h line at 2802.

\subsection{Other Systems}

We are interested high-resolution observations of miclly rotating systems, so that we can apply our spectral imaging techniques. There have been a few flares observed from other, 
n sre slowly rotating RS CVn systems. Linsliy et al. (1958) and Catalano (1980) summarized the parameters of these flares.

\section{Conclusions}

- The observing strategy we use for spectral inilging studies allows us to obtain tineresolved, high-resolution spectra of flares. We are therefore able to measure the flux, width, and wavelength of the flare cmission line protile and the change of each of these properties cluring the flare deciiy. These six paraneters can be used to study line broadening, to measure systematic llows, to determine the flare location, and to derive realistic surface fluxes.

- In order to determine these parianeters well, high-resolution spectria must be obtinined before, during, and after the flire. This has been clone only tivice (the $\mathrm{K}$ star flare un V711 Tau and the G star flare on AK Lac).

- The similar aspects of these two fiures are (1) the flux clecay timescales, (2) the amumit of additional line broadening near Hare peak, (?) at systemalic realshift of the flare emission near flare peak, and (4) a rapid decay of this broatloning.

- The firm' ' aviolet integrated line fluxes from these and ouler flares beliave sinilarly. The transition region lines are enlunced by greater factors and decay nore rapilly tlanu the chomospheric lines.

- AR Lac, V711 Tau, and HD 199178 have been ulsserved on several occasions. A crule estimate of the flare frequency is nne per rutation (i.e. 2 or 3 days), but no flares were observed from AR Lac cluring a 1 day observing run in September 1987.

- High-reschution spectria should be obtained throughout the rotational cycle, not juit during tle flare.

\section{REFERENCES}

Catalano, S. 1986, in Flares: Solar and St: /hrt, Proc. RAL Workshop on Astronomy and Astropliysics. Liiisky, J.L., Neff, J.E., Brown, A., Gross, B.I)., Simon, T., Andrews, A.D., Rodunò, M., and Feldman, P.A. 1988, Astron. Astrophys., in in'ss.

Neff, J.E. 1987, Ph.D. thesis, University of Colorado, Boulder.

Nefl, J.E., Walter, P.'M., Rodonò, M., and I insky, J.L. 1988a, Astron. Astrophys., in press.

Nulf, J.E., Vilhu, O., and Walter, F.M. $16 \mathrm{~b}$, in $A$ Decule of $U V^{\prime}$ Astronomy Will the IUE Satellite, ESA-SP281, p. 291.

Simon, T., Linsky, J.L., and Schiffer, F.!! !9S0, Astrophys. J., 239, 911.

Willir, F.M., Gibson, D.M., Brown, A. Carpenter, K., Linsky, J.L., Rudonò, M., Eyles, C. 1981, Bull. AAS, 16, 896.

Walter, F.M., Neff, J.F., Gibson, D.M., : i. ky, J.L., Rodosio, M., Gary, D.E., and Butler, C.J. 1987, Astron. Astrophys., 186, 211 . 
TIME FROM FLARE ONSET (h)

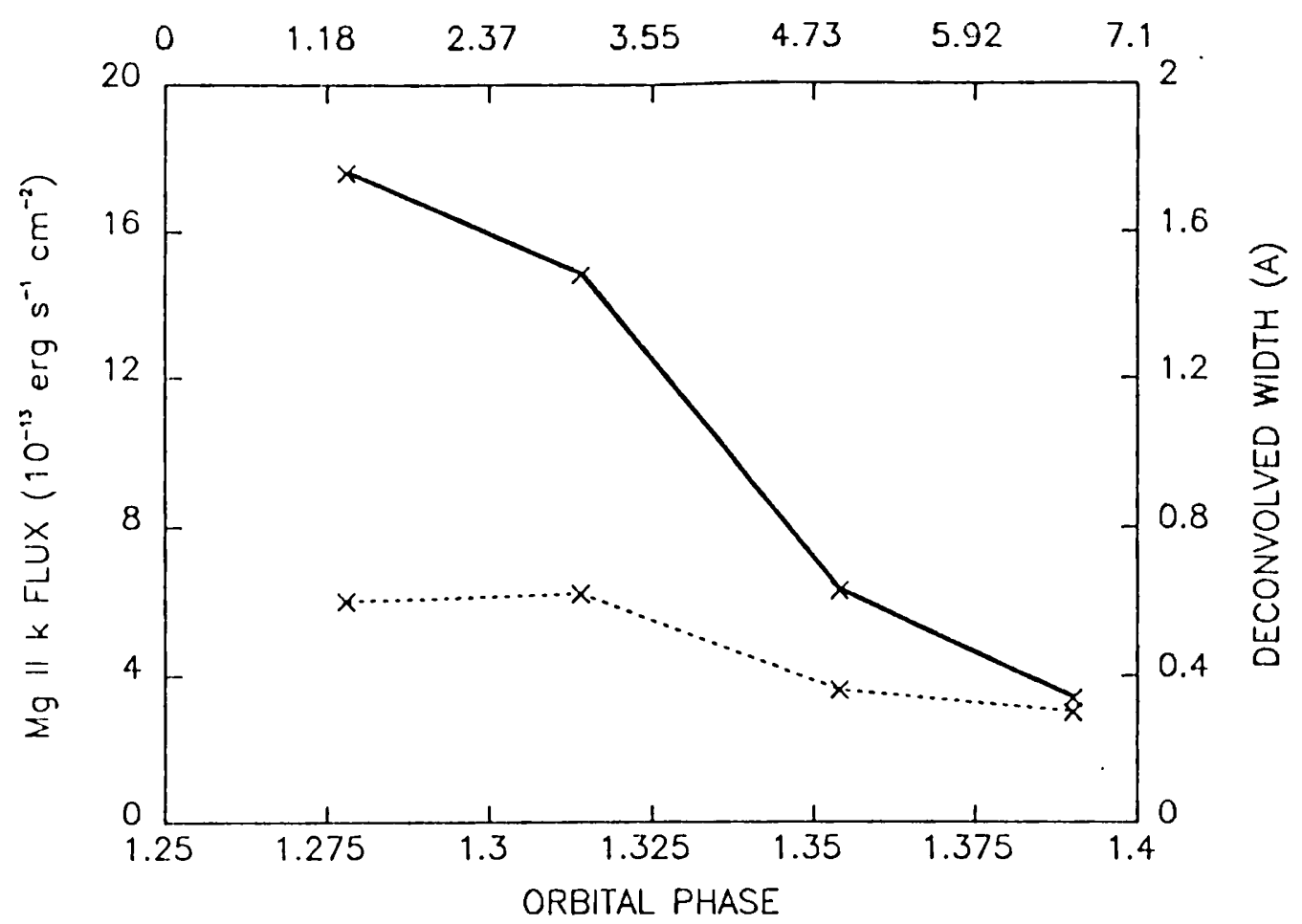

Figure 1: The flux (solid line) and width (dashed line) of the flaring component alone were determined by subtracting the pre-flare $G$ star profile from the observed profile and then fitting the residual emission with a gaussian. The probable phase of flare onset was $\phi=1.255$, during the previons SWP spectrum.

TIME FROM FLARE ONSET (h)

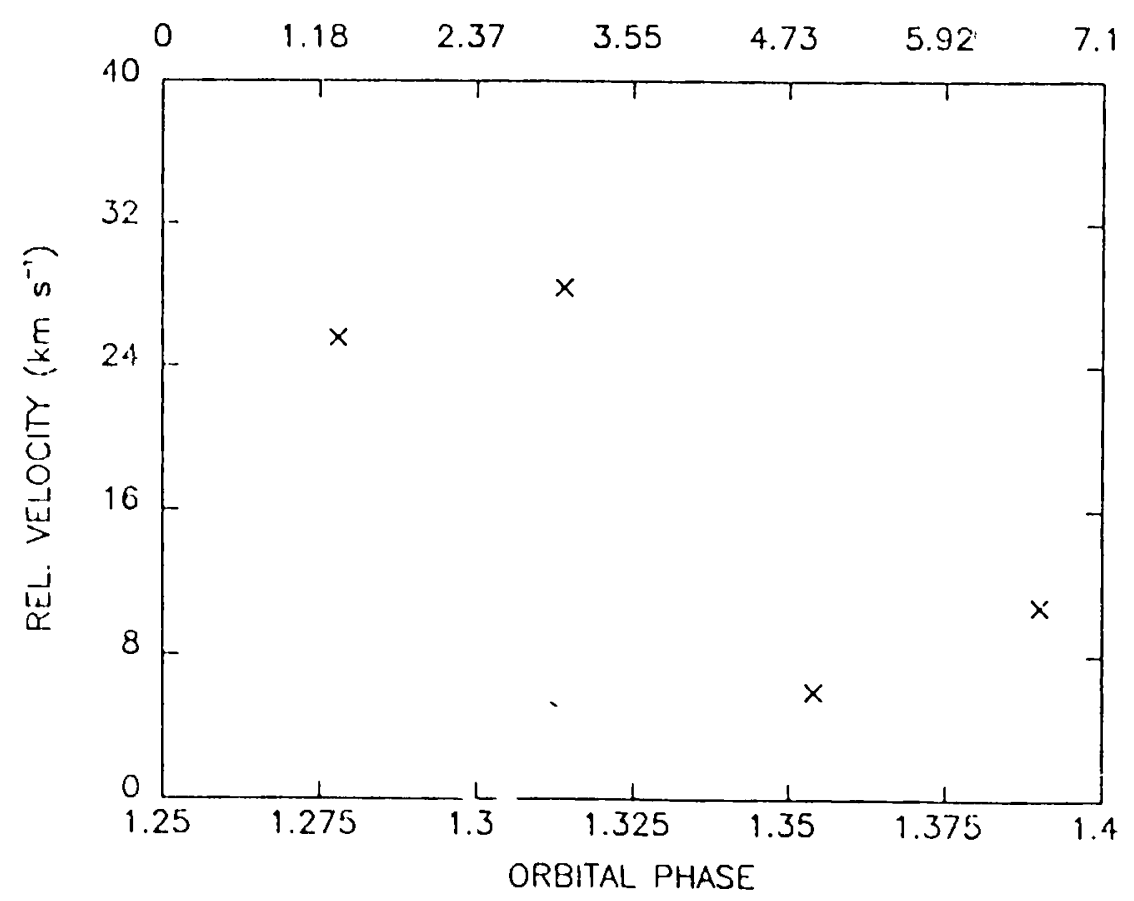

Figure 2: The measured radial velocities of the flare component relutive to the mean radial velocity of the $C$ star. This red-to-blue motion is counter to the rotational (blue-to-red) velocity shift, so the systematic redshift at flare peak is $\sim 30 \mathrm{~km} \mathrm{~s}^{-1}$. 